\title{
How Are Minor Mental Health Problems Perceived by Traditional Chinese Medicine?
}

\author{
Arnulf Kolstad ${ }^{1,2 *}$, Nini Gjesvik ${ }^{3}$ \\ ${ }^{1}$ Department of Psychology, Norwegian University of Science and Technology, Trondheim, Norway \\ ${ }^{2}$ Hangzhou Normal University, Hangzhou, China \\ ${ }^{3}$ Department of Social Work and Health, Norwegian University of Science and Technology, Trondheim, Norway \\ Email: *arnulf.kolstad@svt.ntnu.no
}

Received February 1, 2012; revised April 16, 2012; accepted May 23, 2012

\begin{abstract}
The purpose of this study was to reach a better understanding of how minor mental health problems (MMP) are perceived in China by professionals practicing Traditional Chinese Medicine (TCM) and by well-educated people living in three urban locations. The results derive from interviews with three TCM doctors, three TCM students and eight other students. Psychological problems are separated into two different categories: "serious" and "not serious". MMP are labelled not as disorders or illnesses but looked upon as ordinary problems in daily living or as "heart problems". MMP seem to have less serious consequences according to the Chinese than from a modern Western perspective. "Problems of life" rather than sickness was the category that best summarized perceptions of MMP. TCM professionals' advice to change lifestyle and most Chinese regulate by themselves less serious mental problems. Both lay people and TCM professionals associate serious problems with pathological mental function in a disease perspective. Some reasons for and consequences of these comprehensions are discussed.
\end{abstract}

Keywords: China; Heart Problems; Minor Mental Health Problems; Problems of Life; Traditional Chinese Medicine

\section{Introduction}

In the latter part of the nineteenth Century, China looked to the West and Western science in an effort to modernize the country. The traditional medical practice, "Chinese medicine” as it was called by many Chinese, was abandoned for more than half a century until rejuvenated and reconstructed as Traditional Chinese Medicine (TCM) from 1944 to 1954 [1]. TCM is now used as the international term for "Chinese medicine" and the term used in this article.

In TCM bodily and mental phenomena are explained by the "Five-element or organ theory". The theory focuses on the quality of energy or qi and its balance of yin and yang. Qi comprises psyche and soma [2] and different organs are associated with different psychological functions or emotions; the kidneys with fright and fear, the lungs with sorrow, the heart with joy and happiness, the liver with anger and frustration, the spleen with thinking. Qi, a Taoist concept of energy constitutes the intermediating substance of the dynamic interrelationships between biology and psychology. TCM describes how different forms of qi are created and transmitted in the body and how its emotional aspects generate and

${ }^{*}$ Corresponding author. control psychological energy [1].

TCM is a holistic medical tradition. "The core postulate of the holistic viewpoint is that the notions of mind and body refer to inseparable and mutually dependent aspects of man" [3, p. 159]. This holistic viewpoint is reflected in the labels used in TCM although the conceptualization should not be taken too literally. Some scholars refer to TCM labels as "metaphoric expressions" [4]. Organs such as the liver, heart or spleen do not refer to single physiological entities as in Western medicine but have a metaphorical connotation and represent a circuit of functions and dynamic relationships called zang fu. The TCM knowledge of zang fu signifies the relationships between the inside (physical-mental) and the outside world (nature-social world-heaven) as specified in the theory of the five elements.

The functions and relationship between zang fu in terms of internal organs and the outside world are mediated by $q i$ which also represents the relationship of the two dialectical forces yin and yang. Qi energy circulates in the body and mediates the relationships between inside and outside. The inside refers to a subjective level of mental body; the outside refers to nature and the social world. Mental and physical problems reflect a dysfunctional or not-harmonious balance of the internal rela- 
tionships between mind and body as well as of the external-internal relationships.

"Heart" refers to the function associated with the heart, not the heart simply as an organ. In TCM "heart" combines emotion and mind, regulating spiritual functions, reflected in thinking, speaking, and behaviour. It is the seat of "the main spirit" that controls "the smaller emotional spirits" connected to other organs [2]. Many Chinese terms for "mental" are made up of the radical xin, meaning heart, often translated as "mind", "emotions" or "mentality" [5]. The meaning of xin expresses a basic assumption underlying Chinese epistemology that the heart/emotions/mind and mind/brain are not separated.

"Heart function" in TCM assumes an integration of thinking and regulation of emotions. This heart-mind function and its regulating effect are taken care of by our thinking, in the way we relate to others and how we speak. There is a close connection between thinking, language and heart, and "the tongue is the opening of the heart". Heart stores shen, meaning spirit [2]. The balance of heart-mind is taken into consideration in all sorts of communication, emphasizing what it is appropriate to say, and how thoughts and behaviour are expressed. Unbalanced energy, like too much yang or heat quality, leads to heat in the heart-mind and may result in phlegm, which obstructs the flow of energy (qi) and disturbs the heart-mind function.

Depression has been translated in Chinese as yiyu, with reference to the $y u$ syndrome in TCM. Yu literally means "not flowing, entangled or clogged" or "stagnation" in English [6]. The construct is used for depression and correlates with symptoms like low mood or low energy. Yi reflects stagnation of qi life-energy. The obstruction of energy flow can lead to jingshenbing, meaning spiritual/mental illness/disease, most often translated as psychiatric disorders or mental illness in Western textbooks [2,5]. In TCM textbooks, jingshenbing is a term for medical psychiatry and covers all mental problems from minor to major psychiatric disorders [2,5]. This perspective on jingshenbing, which includes both serious and minor problems, however, does not have the usual sense of meaning among the TCM professionals in this study. They use jingshenbing to denote severe psychiatric illnesses like schizophrenia, bipolar disorder with mania, or grave depression. TCM do not treat such disorders, which are cared for in mental hospitals, not in TCM hospitals. Most of the doctors would say, "I do not have the knowledge nor do I treat this kind of severe mental problem."

The degree of seriousness of most illnesses, including psychiatric disorders, is referred to in terms of function. In the Chinese language, the general designation for mentally disordered behaviour is kuang. In TCM contexts, mental problems are perceived as serious problems if the behaviour is improper or when social functions like the ability to work and communicate are seriously affected. Emphasizing functions or the ability to take part in ordinary social life incorporate a sociological perspective of sickness [7]. Mental problems that seriously affect behaviour, thinking, and working functions should be treated by Western medicine (WM) and not TCM, according to most TCM professionals.

Minor mental health problems are, however, perceived in a quite different manner and are very different from jingshenbing. The least serious problems are often labelled jiaolü zheng in Chinese. Zheng is translated as sign/symptom, an external manifestation, and used in TCM for pathological syndromes [1]. Jiaolü is a buzzword with more than one million hits on Chinese websites [8]. It includes stress, anxiety, and minor depression. The number of hits indicates, however, increasing concern about these less serious problems among the Chinese.

The therapeutic process which is characterized as tiao (attuning, balancing) targets the patient's overall illness condition and experience, including physical and nonphysical aspects of suffering. The TCM doctor focuses on advice and negotiation, aiming to activate a good attitude in order to resolve problems and remain in a job. Adjustment of the patient's perceptions of reality and social relations is salient to the "attuning" process and the doctor's dialogue with the patient as negotiating perspectives on understanding symptoms and functions is crucial from a TCM perspective [9]. This negotiation of meaning, which is intended to activate a good attitude in terms of perceiving and resolving problems and maintain role function; is part of the balancing process in TCM treatment [9]. MMP are not perceived as illnesses in need of treatment by most Chinese but as problems requiring balancing strategies within the scope of daily "life cultivation” [10]. MMP in terms of depression, dysthymia, and psychosomatic problems like digestive symptoms and tiredness are related to energy deficiency and connected with weakness/fatigue/depression.

Several studies within China and among Chinese abroad point to the heavy stigmatization of people with mental disorders [11-15]. Obtaining a psychiatric diagnosis is still something very shameful in China and people can lose their job if so diagnosed. People therefore do not want to visit a psychologist or mental hospital for minor problems since a diagnosis of mental illness may have serious economic and social consequences. One case study points to how fatigue among women in Hong Kong does not signify disease [16]. Instead of being incapacitated, they learn to endure it, and most think it will improve with rest. Some studies illustrate how new patterns of distress such as fatigue are closely related to social structures and economic exigencies [16]. Owing to 
the serious consequences and stigma attached to problems labelled as mental illness there is a tendency in China to label mental problems instead as somatic problems [11,15,17].

The purpose of this study is to reach a better understanding of the perceptions of minor mental problems (MMP) held by well-educated urban Chinese people in contemporary China, both within and outside of the context of traditional Chinese medicine (TCM).

\section{Methods and Materials}

Searches of health science and social science databases (Ovid; PubMed, ICI Web of Science, Amed, PsycInfo) revealed few studies combining the field of Traditional Chinese Medicine and psychology/minor psychiatric disorders in contemporary China. The relatively few English translations of studies of TCM in OVID health databases refer to major psychiatric disorders like depressive psychosis, schizophrenia, and bipolar disorder.

A design for field work including observation and interviews was chosen to investigate the content and meaning of how MMPs are perceived. Both authors have lived in China for some years, have studied traditional Chinese medicine (TCM) and have worked with the mental health services. Data were gathered during a four month field study in China. An interview guide was designed before entering the field. The questions, and their sequencing, opened a process which went from a more general level of disclosing meaning through to a focus on MMPs at a more accurate and specific level. Questions concerning traditional Chinese culture and medicine were left until the end of the interviews. The intention was to avoid the use of Western diagnostic etic concepts, and to access Chinese emic concepts.

The interviews were conducted in English. Instead of using an interpreter who could have made the dialogue more complicated, we looked for people who could make themselves understood in English. We did not require extensive knowledge of English or fluent language skills.

When using an observational method in conjunction with interviews and dialogue the aim is to reveal the most typical (prototypical) attitudes and to find some coherence or consistency between attitudes and cultural traits. These kinds of qualitative studies do not deal with traditional validity and reliability, as in quantitative studies. Validity, in qualitative research also called trustworthiness, refers to whether the results may be regarded as answering the research question. The methodology used in this study represents the analytical or rational tradition in science just as much as empirical traditions. The quotations from the interviews/dialogue are used to illustrate (proto) typical points of view, and the explanations given in the text are not to be tested or verified by the selected quotations or statements. Quotations are used to illustrate the position and arguments given in the running text. This article does not focus on the differences between the 14 people interviewed, since there are too few to make such differences meaningful and because the main aim has been to present prototypical answers.

\subsection{Sampling}

The purposive sampling used in the study is characterized by the selection of information-rich cases for study in depth [18]. Certain sampling criteria, such as being Chinese and living in China, were obviously necessary to elicit a Chinese meaning within a Chinese contemporary context. Three different sites were chosen for fieldwork and interviews. The selection of participants was carried out during the field stay in a way that could be called "casual” or "opportunistic" sampling [18]. Three TCMdoctors, 3 TCM-students and 8 students outside TCM contexts were recruited. There were 6 males and 8 females. Two were from a city in the North, 5 from a city in the West and 7 from a city in the East.

The three TCM doctors who were interviewed worked in three different geographical contexts in China: a city in the West, a city in the North, and a city in the East. They had been working abroad for a period of time, which had influenced their knowledge of MMPs. Two of the doctors were professors. They were interviewed in their work contexts. The doctor from the East was interviewed in a busy clinical hospital, and the doctor from the North at the teachers' office in a TCM university. The third was a young and newly educated TCM doctor, who spent his summer holiday as an apprentice doctor working at the TCM hospital in the West. Five of the participants from outside the TCM context were recruited through an advertisement on a university webpage. The sample consisted of urban people of between 22 and 40 years with an academic background. Although many had grown up in rural areas in a traditional Chinese way, their educational level was high and urban experiences had influenced their attitudes and knowledge. If people within a rural context with less education or older people had been included, the influence of scientific and Western knowledge would have been less obvious and their traditional knowledge might have been stated more clearly.

Words and descriptions like "anxious”, “depressed”, “extremely tired”, or "timid”, “sleep problems”, “eating problems" were used to check the perspectives of meaning in terms of the symptoms of minor mental problems. When listening to their stories, we could ask further questions, but by not being too active in the interview situation we could more easily adapt to the descriptions of their perceived reality and ask questions about their 
conceptions. By taking this phenomenological approach, the interviews necessarily took a long time. When meaning was considered to be sufficiently saturated, we stopped undertaking interviews.

\subsection{Analysis}

The interviews were transcribed verbatim by one of the authors. The meaning of each interview was condensed to the most important words and texts, and statements were themed according to their meaning. When reading the interviews, particular attention was paid to what seemed to be common themes, and to finding the sentences or words the participants had used that could illustrate these themes. Summary response statements were provided for each theme. The research questions guided the process of seeking meaning by providing different contemporary and traditional cultural perspectives on the text.

\subsection{Ethical Reflections}

The interviewees were informed about the aims and purpose of the study, about anonymity and the possibility of further contact after the interview and were given an e-mail address and a Chinese mobile phone number. Everyone gave oral consent to the interview. To protect the anonymity of the persons interviewed, no names were required. The participants were asked if the interview could be tape-recorded, and all except one agreed.

\section{Results and Discussion}

The three TCM doctors who have been practicing abroad said that MMP and stress-related problems such as insomnia, headache, and pain were common among their patients abroad, but not among their Chinese patients in China. "Problems of life" rather than sickness was the category that best summarized perceptions of MMP in China. The term “mental”, a Western concept, emphasizing mental problems as a distinct entity separable from physical problems does not have any meaning in the holistic TCM tradition. Mental problems are perceived as integrated in somatic problems and rely on a holistic acceptance of mental and somatic problems as inseparable.

Somatic pains, neurological dysfunctions like hemiplegia, facial paralysis, and sciatica are common reasons for seeking help at TCM clinics. Some patients have pain problems like headache, shoulder pain and backache; others have menstrual problems or sleep problems, mostly combined with other symptoms. MMP separated from body problems seem to be a very rare reason for contacting a TCM doctor. Doctors at the West TCM hospital were surprised when questioned about their experience or knowledge of MMP. One doctor said: “These kinds of problems are treated in special mental hospitals, not in TCM hospitals."

There are, however, exceptions. One Chinese doctor includes mental aspects and mental problems in her clinical practice and perception of symptoms. She and her assistant seem familiar with the term mental problems and MMP. She considers muscular pain problems to be integrated with mental ones, and pays special attention to the latter when patients have problems with menstruation and sleeping. She uses the five-element theory as well as specific mental points and needling techniques to balance the mental aspects as an integrated part of the treatment.

A young doctor in the West city knows MMP from his practice abroad. He does not believe MMP are common among patients in the TCM hospital, and he supposes MMP to be a rare condition in the West city. The reason for the lack of MMP according to him is a more laidback lifestyle in the West city and he believes this prevents MMP. Therefore MMP are more common among people living in the cities of East China and he associates MMP with life conditions in more urban areas in terms of heavier financial pressures and a busier lifestyle.

\section{1. "Talking Cure"}

According to the TCM doctors Chinese people reduce or solve their minor problems by talking to family and friends, and they therefore do not need professional treatment to the same degree.

In China, people talk with relatives, family, on the Internet, or MSN to solve such problems. Chinese people always want to talk, explain to other people why they feel like this. Foreigners think by themselves, don't talk to others apart from the doctor, and only trust the doctor to talk to. Talking is however always important in the therapy (TCM professor).

TCM doctors emphasize the importance of talking and how the Chinese choose lay contexts, friends and family and foreigners choose professionals for speaking about emotions. People in China prefer unprofessional and private relationships for sharing difficult thoughts and unpleasant emotions. The different choice of professional or lay contexts for expressing problems is the result of deep-seated cultural differences regarding which one shares problems with. This reflects how cultural traditions and knowledge have an impact on perception of problems, symptoms, coping, and therapeutic measures [19-21].

\subsection{Communication in Therapy}

Analysis of communication between TCM doctors and patients with MMP in China describes the doctor's negotiating perspectives in understanding the patient's symp- 
toms and functions [9]. This therapeutic process characterized as tiao (attuning, balancing) targets the patient's overall illness including physical and non-physical aspects of suffering.

Balancing is part of everyday knowledge for most Chinese people, the doctor in the Eastern city says. She attributes lifestyle as the cause and emphasizes lifestyle changes for her patients.

The seeming lack of awareness of MMP in a clinical context among those interviewed in this study indicates that MMP are not regarded as something to be dealt with, if they exist as a health problem at all, in TCM contexts. Somatic problems are given priority. The scant knowledge among TCM doctors, especially those who did not speak English, may indicate that MMP are not part of their applied knowledge. The scarcity of studies on MMP and TCM in China confirms the lack of awareness of MMP among TCM professionals. Mental problems are understood by many doctors as severe mental illness and not regarded as relevant for TCM treatment. The judgment of seriousness is therefore regarded as a problem with both social and economic consequences. The economic and social consequences of a mental illness diagnosis are part of the judgment that influences how symptoms are perceived and labelled by doctors and lay persons. The lack of knowledge and experiences of MMP in TCM may represent a resistance towards regarding and labelling problems as mental since this often results in stigmatization.

\subsection{Serious and Non-Serious Problems}

Some Chinese TCM doctors as well as lay people seem to separate psychological problems into two different categories. A TCM professor in the North city for instance differentiated such problems as "serious" and "not serious" and they were quite separate from each other in both content and function. TCM doctors and lay people often focus on the functional part of a disorder.

Mental problems are very serious if the person can't work, read or think. This is called kuang dian. These people are always excited. They are treated by modern (Western) medicine in mental hospitals, afterwards TCM herbs, decoction and acupuncture. In the patient's body, there are problems with the heart governing the shen. Two pathogenic factors, fire and phlegm stay in the heart, making the heart function change. Fire is yang, always activity, never quietness. Symptoms like red face, red tongue, yellow fur, and a bitter smell... Why phlegm? They can't think, understand who is their mother, father. Phlegm makes it difficult to think step by step.

In TCM we use herbs to clear away the heat and herbs to disperse the phlegm. Needles stimulate and make the patient excited-a nerve massage. If the patient is tired, wanting to sleep, then we will use acupuncture needles (TCM professor).

\subsection{Less Serious Mental Problems}

The doctor in the North city describes how she distinguishes MMP from severe mental disorders, labelling MMP as less serious mental problems. They develop in two stages:

The first step is the liver yang rising, meaning a liver dysfunction. Not many Chinese people would see the doctor at this stage. They would regulate it by food or other things. Jiaolü zheng is the Chinese expression, but the traditional Chinese doctor would say yu zheng. Jiaolü can cause liver function disorder or liver yang rising. In this first less serious step liver functions change. The liver governs the patient's emotions. If the liver qi functions well, it will regulate the balance so that one always feels good and kind.

Liver qi stagnation leads to fire and symptoms such as insomnia, being easily angry, being unable to concentrate, restless walking, unable to focus on one thing. Chinese traditional doctors don't focus on liver yang rising. There are too few doctors in China for this. They would go crazy with too many patients, and the doctor can't solve problems by medicine alone. These problems are connected to lifestyle problems and people must change their lifestyle.

I would tell my patients that they had jiaolü now, and advise them to do some exercises and change their lifestyle. These patients work all the time, stay at home at the computer, don't go outside. Sitting will lead to qi stagnation. I would advise them, for example, to go outside, do some deep breathing exercises so the qi can flow (TCM professor).

The first of these two stages in less serious mental problems are liver dysfunction, which most Chinese, the doctor says, would regulate by themselves, not seeking treatment or a TCM doctor. She points to the importance of balancing mental and physical activity, referring to their interdependent relationship of mutual promotion or control, which is part of the interacting relationships in the five-element theory. These problems are associated with lifestyle and thus are primarily socially explained.

The doctor speaks of how imbalance in the Yin or Yang aspect of liver energy qi leads to symptoms associated with the regulative functions of liver by using the term "control" or "govern" as regards emotions. These terms express the law of movement of the five-element theory of mutually dependent relationships [22]. If the liver's regulative function is in balance, the doctor says one always feels good and kind, and this is associated with feeling satisfied and relaxed. Stress may lead to problems with liver qi, which may lead to problems with 
liver function. Liver dysfunction may cause the liver to lose its controlling or regulative function over anger and the balance of muscular tension/relaxation and emotional problems related to frustration and restlessness and a feeling of tension/stress. Dysfunction of the liver may be associated with anxiety, minor depression, and stress problems as well as premenstrual problems [2].

When the TCM doctor refers to the liver or kidney she refers to different qualities of the life-energy qi connected to the different organ function systems of zang fu. Liver $q i$ thus is of a different quality from kidney $q i$. Kidney in terms of zang fu or organ function does not represent a physical organ, but when translated to a Western perspective it may well carry such a label. When speaking about bodily symptoms and organs, the TCM doctor includes cultural knowledge of corresponding mental functions.

\subsection{Treatment}

If people with liver dysfunction consult her, the female TCM doctor in the Eastern city advises them to change their lifestyle and promotes physical activities to balance the mental functions. Knowledge of balancing is part of everyday knowledge for most Chinese people, the doctor says. She cites lifestyle as a cause of illness and change of lifestyle as a resolution, and she emphasizes lifestyle changes as a personal choice and each individual or family's responsibility. Neither TCM nor the doctor can solve these problems with medicine. These problems are not perceived by most Chinese as illnesses in need of treatment but as problems requiring balancing strategies within the scope of daily life, called "life cultivation" in TCM [10].

The TCM doctor describes the second stage of the less serious mental problems:

The second step I think is heart and spleen function changing; heart and spleen function deficiency. At that stage the patient's tongue will be changing, the colour changes to yellow and the tongue is a little swollen, and always feels heavy; the patient has a poor appetite, and diarrhea or constipation, which means a disorder of the digestive system. When they always feel sick and are always quiet this is more serious. This stage is deficiency; heart function deficiency, not enough qi. Why is the second stage like this? The five-element theory thinks that liver is wood. And the spleen is earth. Wood always limits or damages earth. If wood is strong your earth will be weak. So in the first stage your liver organ is strong, so the second (spleen) will be weak. The second stage will lack energy. Acupuncture makes the patient's energy come out.

Q: But in the second stage, with the heart and spleen dysfunction, would the Chinese not go to see the doctor?
At this stage some people come to see the doctor, because they feel tired (TCM Professor).

Dysfunction of one organ affects other organs according to the five-element theory and disturbs the functional circuit described in the theory of zang fu. Dysfunction of the liver will affect the spleen's function, which is associated with digestion, appetite, and the mental capacity of thinking and studying. In TCM the mental aspect of spleen dysfunction is associated with "overthinking" or grudging [2]. Spleen has a transporting and distributive function of nurture called postnatal $Q i$ to all the other organs. Spleen's “digestive function” may be reflected in the mental function of supporting other organs, empathy, and sorting out what is necessary and what is not [2].

The doctor says some would seek the help of doctors at this stage because they feel tired. MMP are shown in terms of depression, dysthymia, and psychosomatic problems like digestive symptoms and tiredness. The problem is regarded as energy deficiency, which people may perceive as illness connected with weakness/fatigue/depression. It resembles neurasthenia, shenjing shuairuo, which has been described as somatization in mental health studies $[11,12,16]$.

\subsection{Lay Perspective}

Lay perceptions of MMP do not comprehend body-mind interrelationships in the way that TCM does, and they do not refer to the five-element theory. Most of the lay persons were surprised when asked about concepts like liver and whether they thought body organs were related to emotions or mental problems. When a student was asked if she would consult a TCM doctor for minor mental health problems, she answered:

A TCM doctor? You mean a Chinese medicine doctor? We always go to them when we get colds or period pain.

$\mathrm{Q}$ : But not for psychological problems?

No, we don't believe that Chinese medicine doctors can solve psychological problems! A psychological consultant would talk to you about these problems.

But the Chinese doctor would look, smell, take your pulse and ask you about physical things. But I don't think that they can sort out your emotional needs (Student).

All laypersons and even many TCM students considered TCM suitable for somatic problems and illnesses, but not relevant to MMP. The popular knowledge of TCM seems to reflect a dualistic perspective, whereby TCM is regarded as a treatment of bodily symptoms in contradiction to how TCM doctors according to the theory of the five principles understand it.

The lay participants as well as the students of TCM categorize MMP as non-serious problems. All lay participants label MMP as xinli zhang ai. Xinli means psychological and zhang ai means problems or obstacles 
[23]. Zhang ai seems to reflect a perception of MMP as problems in the sense of social/relational obstacles.

Q: What kind of problems would you call minor mental problems?

Minor mental problems are not considered as mental problems. We do not recognize this kind of problem as serious unless it interferes with daily life or family life, like not being able to see or talk to other people, which means xinli bing, disorders of the heart that have to do with relating to others. People would call it xinli zhang $a i$, not mental problems. Mental problems are far more serious (TCM student).

Participants do not regard MMP as serious problems nor do they label MMP as mental problems unless they seriously affect role functioning and relationships with others. This perspective on illness fits Talcott Parson's in terms of role participation [7]. It refers to a common norm of what is considered normal and abnormal [24]. Minor mental health problems, for instance problems in relating to others, are perceived as common or normal difficulties, something everybody has to experience when normal is defined as that which is such as it ought to be [25]. In the most usual sense normal refers to that which is met in the majority of cases of a determined kind, referring either to the average or standard of a measurable characteristic [25]. Perceptions of MMP as non-serious problems reflect a judgment of MMP as normal and quite different from mental problems, which are regarded as real mental or psychiatric problems.

The lay participants use a social explanatory model focusing on social relationships rather than on the holistic model of TCM, which focuses on relationships of body and mind. Most important for lay people is how problems affect daily living and interdependent relationships. If they are not permanently distorted there is no mental problem. Temporary distortions are part and parcel of normal life experiences. Everybody will experience them sometimes, and usually they will disappear by themselves. The focus on relationships and what is going on between people and inside single individuals may be interpreted in terms of collectivism rather than psychological structures in an individualistic perspective.

\section{Severe Mental Problems}

Most participants in this study relate jingshenbing to abnormality and loss of normal functions. Many refer to a serious deviance and to a lack of the sense of shame and loss of dignity reflected in the common norms, but mostly they refer to abnormal cognitive function and behaviour. Some differentiate between less serious problems and, even, suicide in terms of representing a threat only to oneself and mental diseases as representing a threat to others. Some describe severe mental problems as abnormal function or disease of the brain or the nerves.
Both lay people and the TCM professionals associate serious problems with pathological mental function in a disease perspective [24]. Mental illness is an abnormality as regards the common norms of behaviour and cognitive functions, i.e. a commonly agreed standard of normality [25]. Perception of mental illness is thus related to morality and abnormality [24].

\subsection{Summarizing}

Most laymen and TCM professionals perceive MMP not as serious or long-lasting health problems but as common everyday problems. This differs from the Western perspective which considers MMP as psychiatric disorders reflecting abnormal function in terms of thinking and improper behavior [24]. In China "mental problems" is used to describe serious problems or severe psychiatric disorders or illness like psychosis-only these problems are regarded as sickness. MMP are regarded as common everyday problems among TCM professionals as well as among lay people. The focus is not on illness as in the Western conceptualization of minor psychological problems.

Perceptions of MMP are influenced by both traditional cultural knowledge and more modern Western conceptualizations. The labelling of MMP as psychological is influenced by Western knowledge. The label points to thinking or attitude as a way of thinking. It reflects the importance of how problems are perceived. TCM professionals and lay people share a more social perspective on MMP and they emphasize family, relationships, and lifestyle as the cause of such problems and how they should be treated.

To label MMP as mental or as disorder reflects a perception of mental sickness in the sense of serious/major psychiatry. It points to a cultural categorization, which sharply differs between minor and major psychiatric problems and between sickness and the normal problems of life.

Mental illness is perceived as a threat to sense of self, how one is regarded by others, and economic and social conditions. The labels of mental and illness represent a stigma in terms of disqualified status with moral implications. The MMP do not represent such a condition for the Chinese, and people who do have such problems are not seen as being in need of medical or psychological treatment.

Attitudes toward professional help and labelling problems as psychological or strange reflect the categorization of these problems within a daily life perspective. This may be interpreted as resistance to categorizing MMP in terms of sickness in need of professional treatment. This resistance toward perceiving MMP in a Western perspective of sickness may be interpreted as a 
resistance to medicalizing the problems of life as sickness. The sense of strangeness toward psychology and professional help-seeking also express a reluctance to regard such problems from an individualistic perspective in accordance with a collectivistic Chinese culture, which combines practical thinking with pragmatic solutions and a shared view of the world with an emphasis on context [26]. Such life problems are normal, common problems to be shared among friends and not in professional contexts related to a sickness perspective. In China, MMP are given low priority by laymen, doctors, and society alike.

\section{REFERENCES}

[1] X. Cheng, "Chinese Acupuncture and Moxibustion,” Revised Edition, Foreign Language Press, Beijing, 2004.

[2] O. Heyerdahl and N. Lystad, "Traditional Chinese Acupuncture. Its Philosophy and Application in Modern Medicine. Textbook 2,” Norwegian Doctors Acupuncture Courses, Trondheim, 2000.

[3] Z. J. Lipowski, "What does the Word 'Psychosomatic' Really Mean? A Historical and Semantic Inquiry,” Psychosomatic Medicine, Vol. 46, No. 2, 1984, pp. 153-171.

[4] T. Ots, "The Silenced Body-the Expressive Leib: On the Dialectic of Mind and Life in Chinese Cathartic Healing," In: T. J. Csordas, Ed., Embodiment and Experience: The Existential Ground of Culture and Self, Cambridge University Press, New York, 1994, pp. 116136.

[5] B. Flaws and J. Lake, "Chinese Medical Psychiatry: A Textbook and Clinical Manual,” Blue Poppy Press, Boulder, 2004.

[6] S.-M. Ng, C. L. W. Chan, D. Y. F. Ho, Y.-Y. Wong and R. T. H. Ho, "Stagnation as a Distinct Clinical Syndrome: Comparing 'Yu' (Stagnation) in Traditional Chinese Medicine with Depression," British Journal of Social Work, Vol. 36, No. 3, 2006, pp. 467-484. doi:10.1093/bjsw/bcl008

[7] T. Parsons, "Definitions of Health and Illness in the Light of the American Values and Social Structure,” In: E. Jaco and E. Gartley, Eds., Patients, Physicans and Illness: A Resource Book in Behavioral Science and Health, Collier-Macmillan, London, 1979, pp. 165-187.

[8] W. Mogel, “The Oy Oy Oy Show: How Anxiety Harms Student Academic Performance,” 2005.

http://www.wendymogel.com/articles/item/the_oy_oy_o y_show_independent_school_magazine/

[9] Y. Zhang, "Negotiating a Path to Efficacy at a Clinic of Traditional Chinese Medicine," Culture, Medicine and Psychiatry, Vol. 31, No. 1, 2007, pp. 73-100. doi:10.1007/s11013-006-9039-6

[10] X. Wang, "Life Cultivation and Rehabilitation of Traditional Chinese Medicine,” Publishing House of Shanghai
University of Traditional Medicine, Shanghai, 2003.

[11] W.-S. Tseng and D. Y. H. Wu, "Chinese Culture and Mental Health,” Academic Press, Orlando, 1985.

[12] A. Kleinman and T.-Y. Lin, "Normal and Abnormal Behavior in Chinese Culture,” D. Reidel Publishing Company, Dordrecht, 1981.

[13] K. Yip, “Taoistic Concepts of Mental Health: Implications for Social Work Practice with Chinese Communities,” Families in Society, Vol. 86, No. 1, 2006, pp. 35-45.

[14] K. Yip, “Community Mental Health in the People’s Republic of China: A Critical Analysis,” Community Mental Health Journal, Vol. 42, No. 1, 2006, pp. 41-51.

[15] N. Z. Hampton, T. Yeung and C. H. Nguyen, "Perceptions of Mental Illness and Rehabilitation Service in Chinese and Vietnamese Americans,” Journal of Applied Rehabilitation Counseling, Vol. 38, No. 2, 2007, pp. 14-48.

[16] S. Lee, "Fat, Fatigue and the Feminine: The Changing Cultural Experience of Women in Hong Kong," Culture, Medicine and Psychiatry, Vol. 23, No. 1, 1999, pp. 51-77. doi:10.1023/A:1005451614729

[17] G. Parker, G. Gladstone and K. T. Chee, "Depression in the Planet's Largest Ethnic Group: The Chinese," American Journal of Psychiatry, Vol. 158, No. 6, 2001, pp. 857-864.

[18] M. Q. Patton, "Purposeful Sampling I: Qualitative Evaluation and Research Methods,” Sage, London, 1990.

[19] A. Kleinman, "Rethinking Psychiatry: From Cultural Category to Personal Experience,” The Free Press, New York, 1988.

[20] A. Kleinman, "Writing at the Margin: Discourse between Anthropology and Medicine," University of California Press, Berkeley, 1995.

[21] T.-J. Ekeland, “A Diagnosis of Psychiatry Diagnoses,” Impuls, Journal of psychology, Vol. 57, No. 1, 2003, pp. 56-64.

[22] D. Y. Yue, "NACS Conference Chinese Culture and Philosophy: Some Characteristics of Chinese Culture and Its Possible Contributions to the World,” 2007. http://www.nacsorg.com/stockholmconf/characteristics_o f_chinese_culture.htm

[23] Collins, "English-Chinese Chinese-English Dictionary," Foreign Language Teaching and Research Press, Beijing, 2004.

[24] C. G. Helman, “Culture, Health and Illness,” 5th Edition, Hodder Arnold, London, 2007.

[25] G. Canguilhem, “A Critical Examination of Certain Concepts: The Normal, Anomaly and Diesase; the Normal and the Experimental," In: G. Canguilhem, Ed., The Normal, and the Pathological, Zone Books, New York, 1991, pp. 125-149.

[26] R. E. Nisbett, “The Geography of Thought: How Asians and Westerners Think Differently and Why," Nicholas Brealey Publishing, London, Boston, 2005. 